\title{
THE EFFECT OF PHOSPHATE CONCENTRATION AND MIXING TIME TO PHOSPHATE ADSORPTION BY DIFFUSIVE GRADIENT IN THIN-FILM (DGT) BASED IRON METAL- ORGANIC FRAMEWORK (FE-MOF) AS A BINDING AGENT
}

\author{
A. Saefumillah ${ }^{\bowtie}$, A. Zulys, and V. Sintya \\ Department of Chemistry, University of Indonesia, 16424, Indonesia \\ ${ }^{\square}$ Corresponding Author: asep.saefumillah@sci.ui.ac.id
}

\begin{abstract}
In this experiment, it would be developed Fe-Metal Organic Frameworks basis (MOFs) as a binding agent in Diffusive Gradient in Thin Film (DGT) to discover the capacity of phosphate adsorption that is influenced by phosphate concentration and mixing time. Phosphorus $(\mathrm{P})$ is one of the materials that are common used by industrial can induced eutrophication phenomenon. This study will evaluate the effect of mixing time and phosphate concentration in phosphate loading capacity. These parameters can be considered for measuring the capacity of phosphate adsorption. The results of phosphate analysis showed that the \% recovery value of Fe-MOFs in the DGT system was $81.29 \%$. The diffusion coefficient of DGT Fe-MOF was $4.74 \times 10^{-7} \mathrm{~cm}^{2} / \mathrm{s}$. The accumulated phosphate will be increased in a row with time increasing. The capacity of phosphate adsorption optimum in $10 \mathrm{mg} / \mathrm{l}$ phosphate concentration. In the final result, it can be concluded that phosphate will be adsorped optimally using Fe-MOF in DGT.
\end{abstract}

Keywords: Phosphate, DGT, MOF, Binding Agent, Iron

RASĀYAN J. Chem., Vol. 14, No.3, 2021

\section{INTRODUCTION}

Phosphate is one of the materials used in some industry especially in agriculture. ${ }^{1}$ The large amount of phosphate can remain the residual that will become phosphate waste ${ }^{1}$. Phosphate waste had to be a concern due to cause environmental pollution especially decrease water quality and eutrophication phenomenon. ${ }^{1}$ Eutrophication phenomenon is a phenomenon of enrichment nutrition to the ecosystem and one of the major elements from phosphate. ${ }^{2}$ In addition to, phosphate waste cause oxygen to decrease due to acidification and increase of oxygen demand. ${ }^{2}$ For sampling the phosphate, there are some techniques sampling and one of common technique using Diffusive Gradient Technique (DGT). ${ }^{3}$ DGT technique utilizes the permeable diffusive gel layer and a binding agent that is suitable for phosphate adsorption. ${ }^{3}$ The principle of the DGT technique is measuring the concentration of average time during diffusion time and applying Ficks First Diffusion Law. ${ }^{4}$

The critical point to maximize the adsorption and selectivity of the analyte is to determine the suitable binding agent. Li et al 2016 and Xie et al, 2017 studies, stated that one of the binding agents for phosphate is iron (Fe) and it was combined with MOFs can increase the capacity of phosphate adsorption because it had high phosphate selectivity compared with another anion $(\mathrm{Cl}, \mathrm{Br}, \mathrm{N}$, and $\mathrm{S})$. Iron $(\mathrm{Fe})$ is available in an environment so it can be easy to do this modification. ${ }^{5,6}$

Latest, there is a new model to develops the porous of material. The model is Metal-Organic Frameworks (MOFs) that have some advantages to increase the capacity of phosphate adsorption due to they have high porosity and large surface area ${ }^{8,9}$ MOFs characterizations can be adjusted by metal ions selection and organic bridging ligands. ${ }^{8-10}$ Another of MOFs advantages is they have good stability and adsorption of water pollutant.$^{7-9}$ Beside that, MOFs also has another advantage become a new role model in photocatalytic research. ${ }^{11}$ So, in the final result, we can evaluate how the effect of phosphate concentration and mixing time on phosphate adsorption by optimizing modification of Fe-MOF in the DGT technique. 


\section{Material}

\section{EXPERIMENTAL}

Ferric Chloride Hexahydrate, Terephtalic Acid, N,N-dimetilformanide, Acrylamide 40\%, N,N'metylenbisacrilamide $1 \%, \mathrm{~N}, \mathrm{~N}, \mathrm{~N}^{\prime}, \mathrm{N}^{\prime}$ - tetrametiletilendiamin $99 \%$, Persulphate, membrane filter (cellulose nitrate), Potassium phosphate monobasic, Sodium Hydroxide, Sodium Nitrate, Sodium Hydrogen Carbonate, Sodium Sulphate, Sulphuric acid, Vitamin C, potassium antimonyl tartrate, ammonium heptamolybdate tetrahydrate, purified water.

\section{Methods}

This modification utilizes the solvothermal method. Mix 1.35 gram of Ferric Chloride Hexahydrate and 0.412 gram of Terephtalic Acid were dissolved in $60 \mathrm{ml} \mathrm{N}, \mathrm{N}$-dimetilformanide and stir for 30 minutes. The solution was transferred into Teflon lined autoclave and heated up to $120^{\circ} \mathrm{C}$ for 20 hours. The product washed using N,N-dimetilformanide. And then the solid was collected by centrifuge and dried at $60{ }^{\circ} \mathrm{C}$. Then, the $10 \mathrm{mg}$ of Fe-MOF crystals mix with $20 \mu \mathrm{L}$ of N,N,N',N'- tetrametiletilendiamin $99 \%$ and $70 \mu \mathrm{L}$ of persulphate $10 \%$ and mold in a square glass then heated in $60{ }^{\circ} \mathrm{C}$ for 45 minutes. After binding gel synthesized, continue with diffusive gel synthesized. Firstly, synthesize the solution gel by mixing Acrylamide $40 \%$ and N,N'-metylenbisacrilamide $1 \%$. Then, take $10 \mathrm{ml}$ of solution gel, $20 \mu \mathrm{L}$ of N,N,N',N'tetrametiletilendiamin $99 \%$ and $70 \mu \mathrm{L}$ of persulphate $10 \%$ and mold in a square glass then heated in $60^{\circ} \mathrm{C}$ for 45 minutes to synthesize diffusive gel.

\section{General Procedure}

The experimental design to measure diffusion coefficient was to optimize Fe-MOF DGT disk in $100 \mathrm{~mL}$ of $10 \mathrm{mg} / \mathrm{L}$ P solution for 4 to 26 hours. The P uptake efficiency of Fe-MOF DGT also optimizes P solution concentration ranging from $1-10 \mathrm{mg} / \mathrm{L}$. The effect of mixing time was designed for 4 to 26 hours. The effect of phosphate concentration was also investigated by optimize the phosphate loading capacity ranging from $5 \mathrm{mg} / \mathrm{L}$ to $15 \mathrm{mg} / \mathrm{L}$.

\section{Detection Method}

The instrument used to detect the MOF formation was X-Ray diffraction (XRD). Then, to examine the surface area, morphology, and functional group is SEM-EDX, Brunauer-Emmet-Teller (BET) analysis, and Fourier Transform Infrared (FTIR) respectively. The capacity of phosphate adsorption in Fe-MOF DGT will be measured by Spectrophotometry Ultraviolet-Visible using ammonium molybdate at wavelength $713.5 \mathrm{~nm}$.

\section{Identification of Fe-MOF Crystals}

\section{RESULTS AND DISCUSSION}

Firstly, Fe-MOF crystals are identified using a bioimaging microscope to know the distribution of crystals, then continue using XRD to examine the crystals phase, and lastly utilize the FTIR to ensure the functional group of MOF exists (Fig.-1).

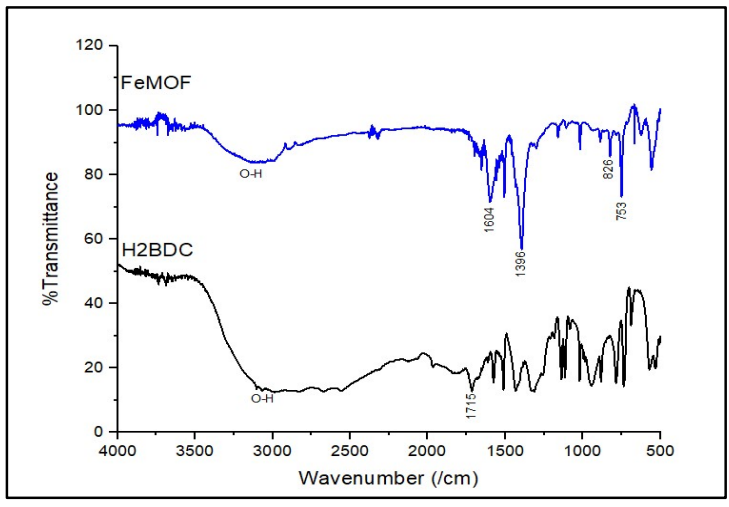

(a)

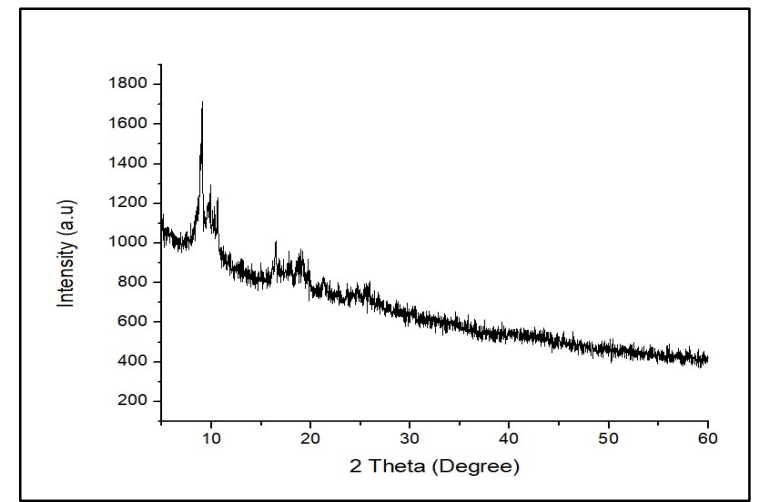

(b) 


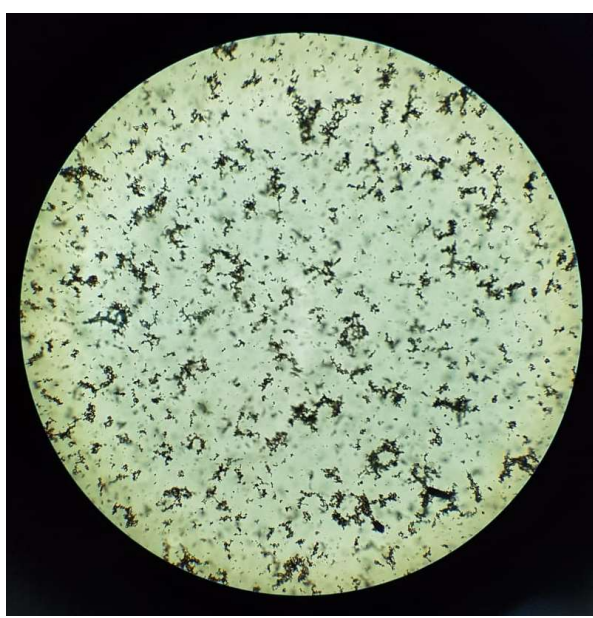

(c)

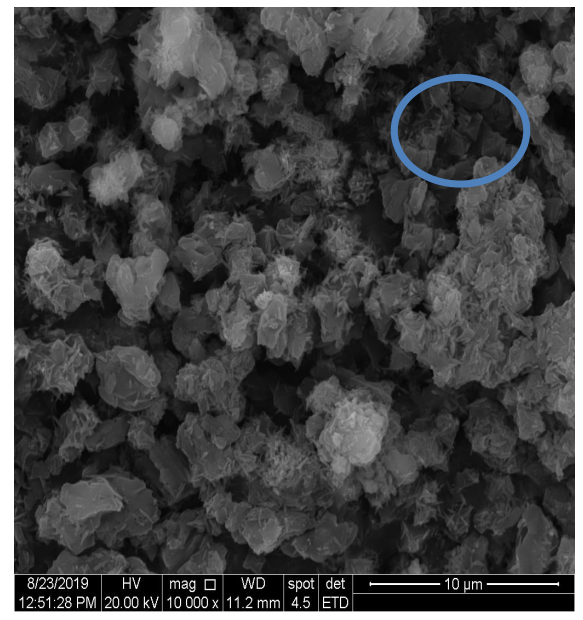

(d)

Fig.-1: Identification of Fe-MOF: (a) FTIR Spectrum, (b) XRD Pattern, (c) Bioimaging Microscope Image and (d) SEM Image

In Fig.-1a (FTIR), there is a unique functional group of MOF. There is benzene-carboxylic main group attributed with $\mathrm{C}=\mathrm{O}$ bond in the carboxylic group and aromatic carbon group $\mathrm{C}-\mathrm{C}$ vibrational mode at $1604 \mathrm{~cm}^{-1}$ and $1396 \mathrm{~cm}^{-1}$ wavenumber respectively. In Fig.-1b (XRD) shows the formation of the crystals exists. It is evidenced by certainty and repetition of crystals structure in 2 theta position at 5.0-10.9 degree and 15.0-20 degree respectively. In XRD result also inform that MOF has high crystallinity due to there is a high-intensity sharp peak. The surface area is only $61.33 \mathrm{~m}^{2} / \mathrm{g}$ examined by BET due to the characteristic of $\mathrm{FeCl}_{3} .6 \mathrm{H}_{2} \mathrm{O}$ is amorf. In Fig.-1c (bioimaging microscope), the distribution of Fe-MOF is homogeny and figure 1d (SEM) shows the morphology is like a prism with a rude surface.

\section{Elution Value of Phosphate Adsorption}

After identified the Fe-MOF crystals, measure the capacity of dissolved reactive phosphate in Fe-MOF binding gel. The binding gel was eluted using $10 \mathrm{ml}$ of $\mathrm{H}_{2} \mathrm{SO}_{4} 1 \mathrm{M}$ for 20 hours then neutralized using $\mathrm{NaOH} 1 \mathrm{M}$. Firstly, perform the eluent optimation by varying the $\mathrm{H}_{2} \mathrm{SO}_{4}$ concentration from $0.025 \mathrm{M}$ to 1 M. The results show the optimum eluent for phosphate analysis is $\mathrm{H}_{2} \mathrm{SO}_{4} 1 \mathrm{M}$ with $81.29 \%$ elution value. This stage aims to define the capacity of phosphate adsorption.

Table-1 : Elution Value of Phosphate Adsorption

\begin{tabular}{c|c|c|c|c|c}
\hline Eluent & Absorbance & Dilution Factor & C $_{\text {Elution }}(\mathrm{ppm})$ & \% Elution & Rata-rata \\
\hline $\mathrm{H}_{2} \mathrm{SO}_{4} 1 \mathrm{M}(1)$ & 0.016 & 20 & 1.037509976 & 81.25 & \\
\cline { 1 - 5 } $\mathrm{H}_{2} \mathrm{SO}_{4} 1 \mathrm{M}(2)$ & 0.017 & 20 & 1.197126895 & 68.18 & \multirow{2}{*}{$81.29 \%$} \\
\hline $\mathrm{H}_{2} \mathrm{SO}_{4} 1 \mathrm{M}(3)$ & 0.018 & 20 & 1.356743815 & 94.44 & \\
\hline
\end{tabular}

\section{Diffusion Coefficient}

After the elution value was obtained, continue to measure the diffusion coefficient (D). The D value is obtained from linearity regression of time (second) and mass of accumulated P (ng) in Fe-MOF DGT. The design of this experiment was Fe-MOF DGT soak in $13 \mathrm{mg} / \mathrm{L}$ DRP solution for 4 to 26 hours. From linearity regression in Fig.-2 shows the $\mathrm{R}^{2}$ value is 0.9921 so $\mathrm{D}$ value is obtained $4.74 \times 10^{-7} \mathrm{~cm}^{2} \mathrm{~s}^{-1}$. This result lower than Zhang et all, 1998 due to the structure of $\mathrm{FeCl}_{3} \cdot 6 \mathrm{H}_{2} \mathrm{O}$ are amorf and low porosity. In Fig.-2 also inform the effect of increasing time leads to an increase in the capacity of loading phosphate. The optimum time for maximum accumulated phosphate is for 26 hours.

\section{The Capacity of Loading Phosphate in Fe-MOF DGT}

The phosphate adsorption from aqueous solution onto Fe-based MOF as a function of mixing time is shown in Fig.-3. The adsorption capacity increased in a row of increasing time. In the first 4 hours, the adsorption 


\section{RASĀYAN J.Chem.}

Vol. 14 | No. 3 |1724-1728| July - September | 2021

of capacity Fe-MOF was reached $1.6 \mu \mathrm{g}$. The adsorption of capacity continued to increase with increasing time. In the 26 hours, the capacity of phosphate adsorption was $14.5 \mu \mathrm{g}$. This situation because the binding agent was in phosphate solution for a long time so it had a chance to adsorb more phosphate.

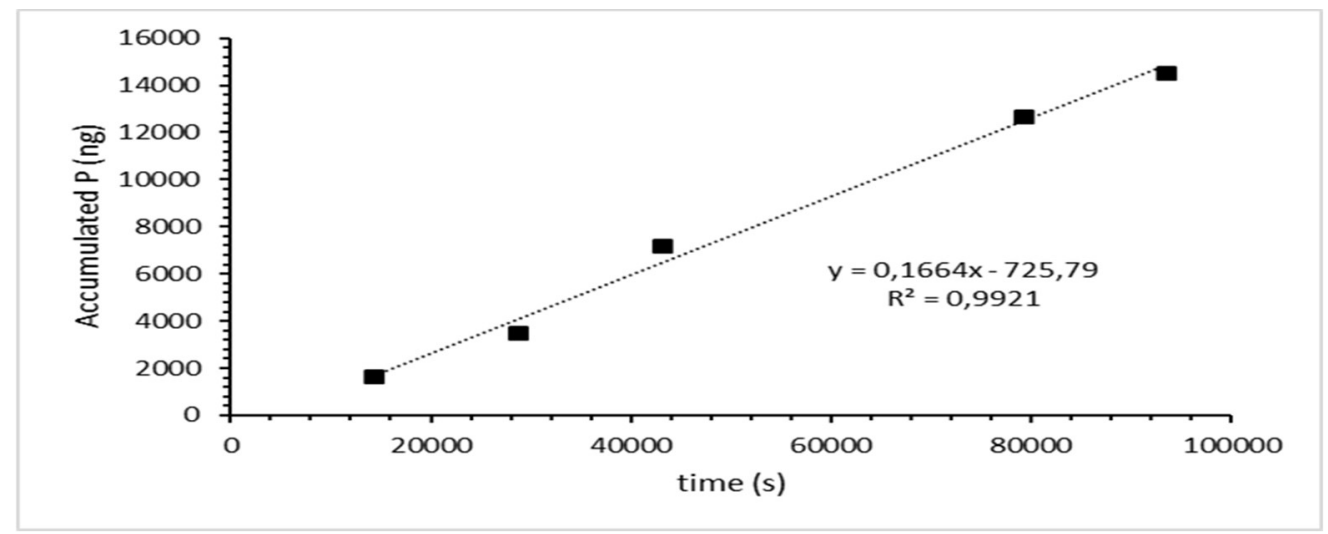

Fig.-2: Linearity Graph of Time and Accumulated Phosphate

In Fig.-4, we can also see the effect of phosphate concentration variation. In the first concentration $5 \mathrm{mg} / \mathrm{L}$ the adsorption of capacity Fe-MOF was reached $3.08 \mu \mathrm{g}$ and then until concentration $10 \mathrm{mg} / \mathrm{L}$ had a constant value. This situation means that the capacity of Fe-MOF in phosphate adsorption optimum is 10 $\mathrm{mg} / \mathrm{L}$.

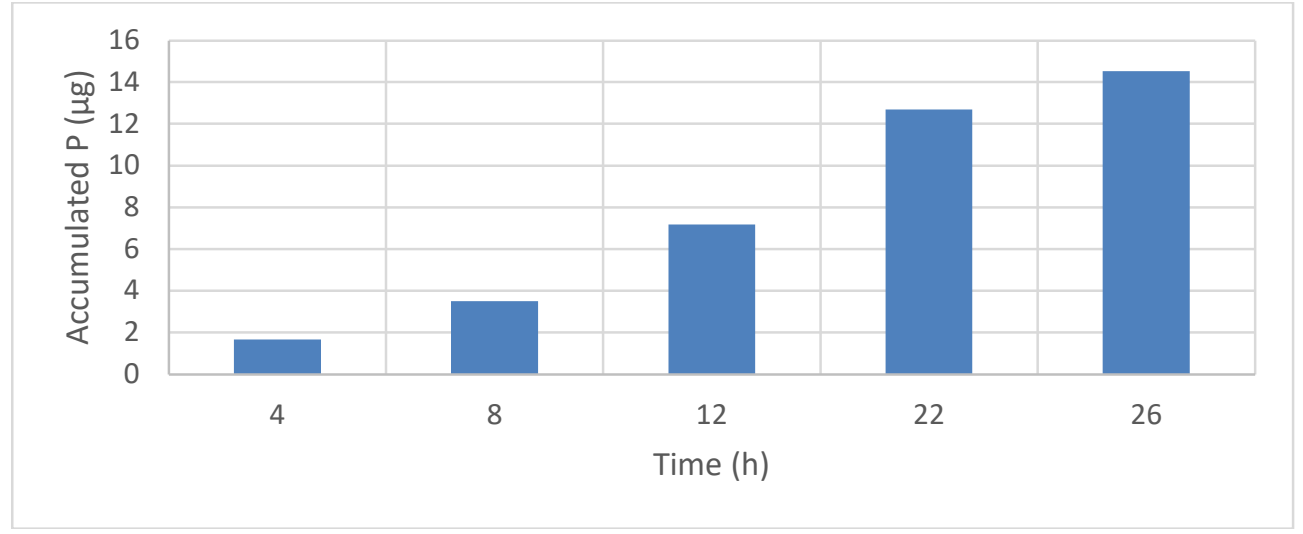

Fig.-3: The Effect of Mixing Time

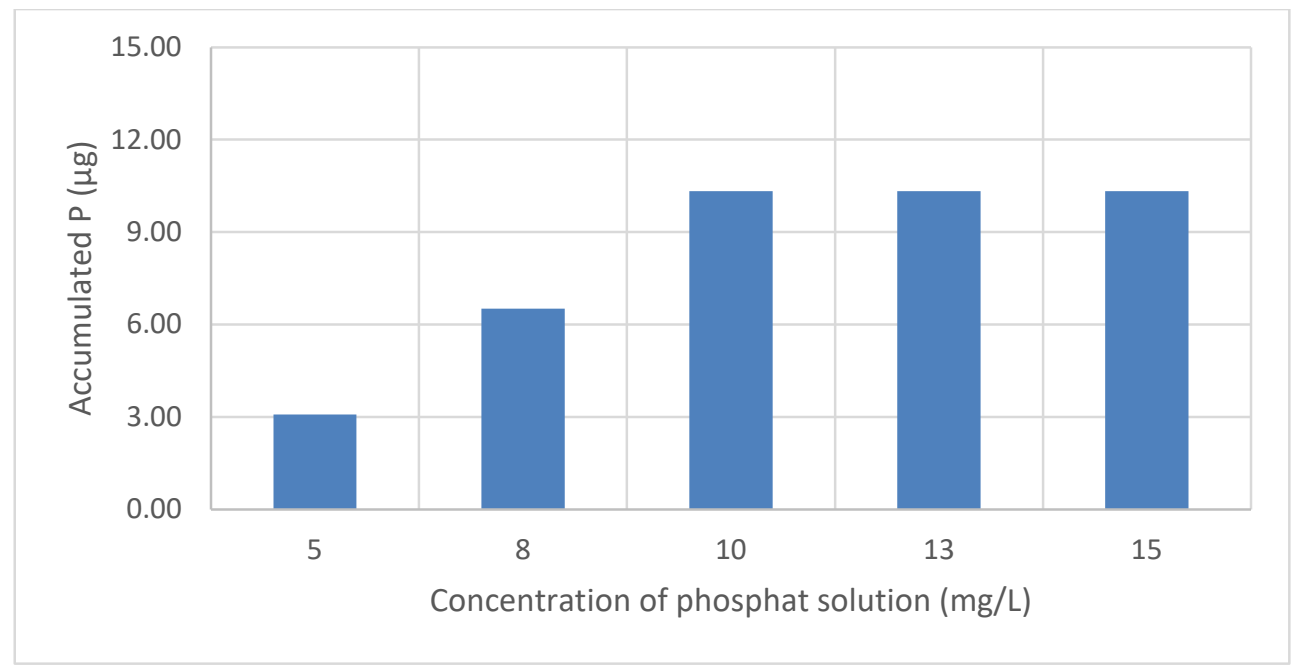

Fig.-4: The Effect of Phosphate Concentration Variation 
RASĀYAN J. Chem.

Vol. 14 | No. 3 |1724-1728| July - September | 2021

\section{CONCLUSION}

This experiment was successful to synthesize Fe-MOF by solvothermal methods in the DGT technique. Mixing time and phosphate concentration influence the capacity of loading phosphate. The final result was showed modification of Fe-MOF in the DGT technique had phosphate adsorption activity.

\section{ACKNOWLEDGEMENT}

We gratefully acknowledge funding by the University of Indonesia Competitive Research Grant (PITTA A 2019) for conducting research.

\section{REFERENCES}

1. Karageorgiou Konstantinos, Maximos Paschalis, Georgios N. Anastassakis, Journal of Hazardous Materials, A139, 447(2007), https://doi.org/10.1016/j.jhazmat.2006.02.038

2. O'Sullivan P.E, International Journal of Environmental Studies, 47(3-4), 173(2007), https://doi.org/10.1080/00207239508710958

3. Cusnir Ruslan, Philipp Steinmann, Francois Bochud, Pascal Froidevaux, Environmental Science \& Technology, 48, 10829(2014), https://doi.org/10.1021/es501149v

4. Ding Shiming, Yan Wang, Liping Zhang, Lv Xu, Mengdan Gong, Chaoseng Zhang, Royal Society of Chemistry, 6, 88144 (2016), https://doi.org/10.1039/c6ra19677b

5. Li Yan, Qiying Xie, Qian Hu, Chengping Li, Zhangjie Huang, Xiangjun Yang, Hong Guo, Scientific Report, 6, 30651(2016), https://doi.org/10.1038/srep30651

6. Xie Qiying, Yan Li, Zhaoling Lv, Hang Zhou, Xiangjun Yang, Jing Chen, Hong Guo, Scientific Report 7, 3316(2017), https://doi.org/10.1038/s41598-017-03526-X

7. Zhao Bei, Yu Zhang, Xiaomin Dou, Hongying Yuan, dan Min Yang, Water Science \& Technology 72(12), 2179(2015), https://doi.org/10.2166/wst.2015.438

8. Furukawa Hiroyasu, Kyle E. Cordova, Michael O'Keeffe, Omar M. Yaghi, The Chemistry and Applications of Metal-Organic Frameworks, 341, 1230444(2013), https://doi.org/10.1126/science.1230444

9. Qin Wenxiu, Gu Yue, Wang Guozhong, Wu Tianxing, Zhang Haimin, Xiaoxian Tang, Yunxia Zhang, Huijun Zhao, Waters Research, 147, 223(2018), https://doi.org/10.1016/j.watres.2018.10.003

10.Zhou Hong Cai, Jeffrey R. Long, Omar M. Yaghi, Metal Organic Frameworks, American Chemical Society, 112, 673(2012), https://doi.org/10.1021/cr300014x

11. Thi Minh Thanh Huynh, Nguyen Thi Thanh Tu, Nguyen Phi Hung, Tran Ngoc Tuyen, Tran Xuan Mau, Dinh Quang Khieu, Journal of Porous Materials, 26, 1699(2019), https://doi.org/10.1007/s10934-019$\underline{00767-1}$

[RJC-5756/2020] 